\title{
Test of lepton flavor violation with Ke2 decay at KLOE
}

\author{
Barbara Sciascia ${ }^{* \dagger}$ \\ $L N F-I N F N$ \\ E-mail: barbara.sciasciadlnf.infn.it
}

We present a precise measurement of the ratio $R_{K}=\Gamma(K \rightarrow e v(\gamma)) / \Gamma(K \rightarrow \mu v(\gamma))$ performed with the KLOE detector. The results are based on data collected at the Frascati $e^{+} e^{-}$collider DA $`$ NE for an integrated luminosity of $2.2 \mathrm{fb}^{-1}$. We find $R_{K}=\left(2.493 \pm 0.025_{\text {stat }} \pm 0.019_{\text {syst }}\right) \times$ $10^{-5}$, in agreement with the Standard Model expectation. This result is used to improve constraints on parameters of the Minimal Supersymmetric Standard Model with lepton flavor violation.

2009 KAON International Conference KAONO9,

June 09 - 122009

Tsukuba, Japan

${ }^{*}$ Speaker.

${ }^{\dagger}$ for the KLOE Collaboration: F. Ambrosino, A. Antonelli, M. Antonelli, F. Archilli, P. Beltrame, G. Bencivenni, C. Bini, C. Bloise, S. Bocchetta, F. Bossi, P. Branchini, G. Capon, D. Capriotti, T. Capussela, F. Ceradini, P. Ciambrone, E. De Lucia, A. De Santis, P. De Simone, G. De Zorzi, A. Denig, A. Di Domenico, C. Di Donato, B. Di Micco, M. Dreucci, G. Felici, S. Fiore, P. Franzini, C. Gatti, P. Gauzzi, S. Giovannella, E. Graziani, M. Jacewicz, V. Kulikov, G. Lanfranchi, J. Lee-Franzini, M. Martini, P. Massarotti, S. Meola, S. Miscetti, M. Moulson, S. Müller, F. Murtas, M. Napolitano, F. Nguyen, M. Palutan, A. Passeri, V. Patera, P. Santangelo, B. Sciascia, A. Sibidanov, T. Spadaro, M. Testa, L. Tortora, P. Valente, G. Venanzoni, and R. Versaci. 
The decay $K^{ \pm} \rightarrow e^{ \pm} V$ is strongly suppressed, $\sim$ few $\times 10^{-5}$, because of conservation of angular momentum and the vector structure of the charged weak current. It therefore offers the possibility of detecting minute contributions from physics beyond the Standard Model (SM). This is particularly true of the ratio $R_{K}=\Gamma(K \rightarrow e v) / \Gamma(K \rightarrow \mu v)$ which, in the SM, is calculable without hadronic uncertainties [1, 2]. Physics beyond the SM, for example multi-Higgs effects inducing an effective pseudo-scalar interaction, can change the value of $R_{K}$. It has been shown in Ref. [3] that deviations of $R_{K}$ of up to a few percent are possible in minimal supersymmetric extensions of the SM (MSSM) with non vanishing $e-\tau$ scalar lepton mixing. To obtain accurate predictions, the radiative process $K \rightarrow e v \gamma\left(K_{e 2 \gamma}\right)$ must be included. In $K_{e 2 \gamma}$, photons can be produced via internalbremsstrahlung (IB) or direct-emission (DE), the latter being dependent on the hadronic structure. Interference among the two processes is negligible [4]. The DE contribution to the total width is approximately equal to that of IB [4] but is presently known with a 15\% fractional accuracy [5].

$R_{K}$ is defined to be inclusive of $\mathrm{IB}$, ignoring however DE contributions. A recent calculation [2], which includes order $e^{2} p^{4}$ corrections in chiral perturbation theory $(\chi \mathrm{PT})$, gives:

$$
R_{K}=(2.477 \pm 0.001) \times 10^{-5} .
$$

$R_{K}$ is not directly measurable, since IB cannot be distinguished from DE on an event-by-event basis. Therefore, in order to compare data with the SM prediction at the percent level or better, one has to be careful with the DE part. We define the rate $R_{10}$ as:

$$
R_{10}=\Gamma\left(K \rightarrow e v(\gamma), E_{\gamma}<10 \mathrm{MeV}\right) / \Gamma(K \rightarrow \mu \nu) .
$$

Evaluating the IB spectrum to $\mathscr{O}\left(\alpha_{\mathrm{em}}\right)$ with resummation of leading logarithms, $R_{10}$ includes $93.57 \pm 0.07 \%$ of the IB,

$$
R_{10}=R_{K} \times(0.9357 \pm 0.0007) .
$$

The DE contribution in this range is expected to be negligible. $R_{10}$ is measured without photon detection. Some small contribution of DE is present in the selected sample. In particular, DE decays have some overlap with the IB emission at high $p_{e}$. We have also measured [7] the differential width $\mathrm{d} R_{\gamma} / \mathrm{d} E_{\gamma}$ for $E_{\gamma}>10 \mathrm{MeV}$ and $p_{e}>200 \mathrm{MeV}$ requiring photon detection, both to test $\chi \mathrm{PT}$ predictions for the $\mathrm{DE}$ terms and to reduce possible systematic uncertainties on the $R_{10}$ measurement.

\section{Selection of leptonic kaon decays}

$K^{ \pm}$decays are signaled by the observation of two tracks with the following conditions. One track must originate at the interaction point (IP) and have momentum in the interval $\{70,130\}$ $\mathrm{MeV}$, consistent with being a kaon from $\phi$-decay. The second track must originate at the end of the previous track and have momentum larger than that of the kaon, with the same charge. The second track is taken as a decay product of the kaon. The point of closest approach of the two tracks is taken as the kaon decay point $\mathrm{D}$ and must satisfy $40<r_{\mathrm{D}}<150 \mathrm{~cm},\left|z_{\mathrm{D}}\right|<80 \mathrm{~cm}$. The geometrical acceptance with these conditions is $\sim 56 \%$, while the decay point reconstruction efficiency is $\sim 51 \%$. From the measured kaon and decay particle momenta, $\mathbf{p}_{K}$ and $\mathbf{p}_{\mathrm{d}}$, we compute the squared mass $m_{\ell}^{2}$ of the lepton for the decay $K \rightarrow \ell v$ assuming zero missing mass:

$$
m_{\ell}^{2}=\left(E_{K}-\left|\mathbf{p}_{K}-\mathbf{p}_{\mathrm{d}}\right|\right)^{2}-\mathbf{p}_{\mathrm{d}}^{2} .
$$


The distribution of $m_{\ell}^{2}$ is shown in Fig. 1 left panel, upper curve, from MC simulation. The muon peak is quite evident, higher masses corresponding to non leptonic and semileptonic decays. No signal of the $K \rightarrow e v\left(K_{e 2}\right)$ decay is visible. The very large background around zero mass is the tail of the $K \rightarrow \mu \nu\left(K_{\mu 2}\right)$ peak, due to poor measurements of $p_{K}, p_{\mathrm{d}}$ or the decay angle, $\alpha_{K \mathrm{~d}}$. The expected signal from $K_{e 2 \gamma}$ is also shown in Fig. 1 left, lower curves, separately for $E_{\gamma}>10$ and $<10 \mathrm{MeV}$. The expected number of $K_{e 2}$ decays in the sample is $\sim 30,000$. A background rejection of at least 1000 is necessary, to obtain a $1 \%$ precision measurement of $\Gamma\left(K_{e 2}\right)$, with an efficiency of $\sim 30 \%$.
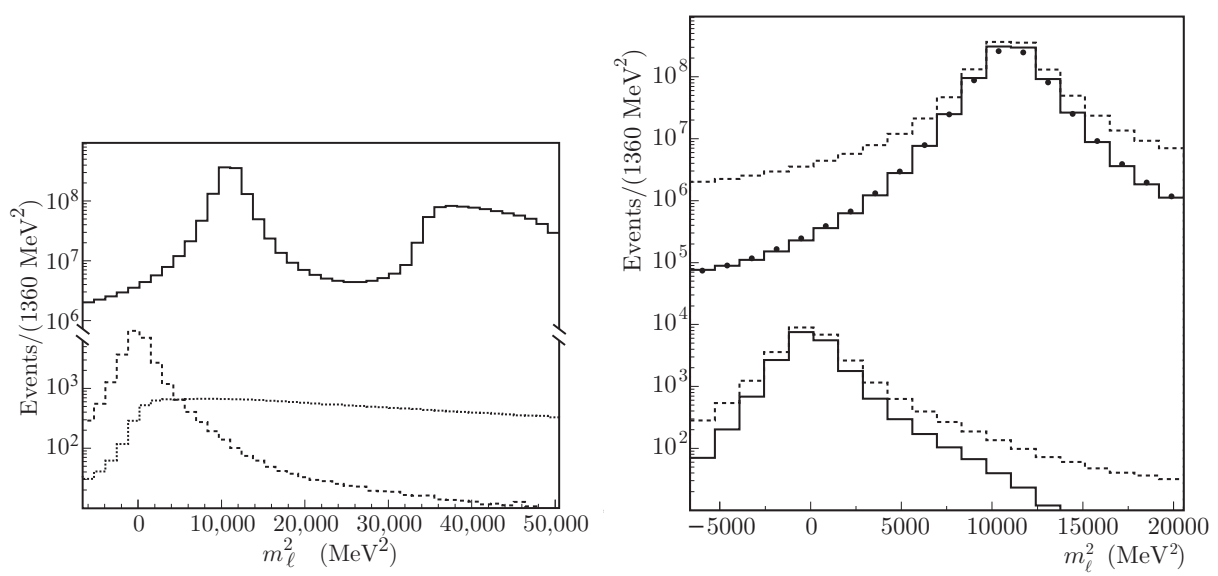

Figure 1: Left: MC distribution of $m_{\ell}^{2}$, solid line. The contribution of $K_{e 2 \gamma}$ with $E_{\gamma}<10 \mathrm{MeV}$ ( $>10 \mathrm{MeV}$ ) is shown by the dashed (dotted) lines. Right: $m_{\ell}^{2}$ spectrum before (dashed) and after (solid) quality cuts for MC $K_{\mu 2}$ (upper plots)and $K_{e 2}$ with $E_{\gamma}<10 \mathrm{MeV}$ (lower plots). Black dots represent data after quality cuts.

The kinematics of the two-body decay $\phi \rightarrow K^{+} K^{-}$provides an additional measurement of $p_{K}$ with $\sim 1 \mathrm{MeV}$ resolution, comparable with that from track reconstruction. We require the two $p_{K}$ determinations to agree within $5 \mathrm{MeV}$. Further cuts are applied to the daughter track. Resolution of track parameters is improved by rejecting badly reconstructed tracks, i.e., with $\chi^{2}$ (track fit) $/ \mathrm{ndf}>$ 7.5. Finally, using the expected errors on $p_{K}$ and $p_{\mathrm{d}}$ from tracking, we compute event by event the error on $m_{\ell}^{2}, \delta m_{\ell}^{2}$. The distribution of $\delta m_{\ell}^{2}$ depends slightly on the opening angle $\alpha_{K d}$, which in turn has different distribution for $K_{e 2}$ and $K_{\mu 2}$. Events with large value of $\delta m_{\ell}^{2}$ are rejected: $\delta m_{\ell}^{2}<\delta_{\max }$, with $\delta_{\max }$ defined as a function of $\alpha_{K d}$, to equalize the losses due to this cut for $K_{e 2}$ and $K_{\mu 2}$. The effect of quality cuts on $m_{\ell}^{2}$ resolution is shown in Fig. 1, right. The background in the $K_{e 2}$ signal region is effectively reduced by more than one order of magnitude with an efficiency of $\sim 70 \%$ for both $K_{e 2}$ and $K_{\mu 2}$.

Information from the EMC is used to improve background rejection. To this purpose, we extrapolate the secondary track to the EMC surface and associate it to a nearby EMC cluster. This requirement produces a signal loss of about $8 \%$. Energy distribution and position along the shower axis of all cells associated to the cluster allow for $e / \mu$ particle identification. For electrons, the cluster energy $E_{\mathrm{cl}}$ is a measurement of the particle momentum $p_{d}$, so that $E_{\mathrm{cl}} / p_{d}$ peaks around 1 , while for muons $E_{\mathrm{cl}} / p_{d}$ is on average smaller than 1 . Moreover, electron clusters can also be distinguished from $\mu$ (or $\pi$ ) clusters by exploiting the granularity of the EMC. All useful information about shower profile and total energy deposition are combined with a 12-25-20-1 structure neural 
network trained on $K_{L} \rightarrow \pi \ell \nu$ and $K_{\mu 2}$ data, taking into account variations of the EMC response with momentum and impact angle on the calorimeter. The distribution of the neural network output, $N N$, for a sample of $K_{L} \rightarrow \pi e v$ events is shown in Fig. 2 left, for data and MC. Additional separation has been obtained using time of flight information. The data distribution of $N N$ as function of
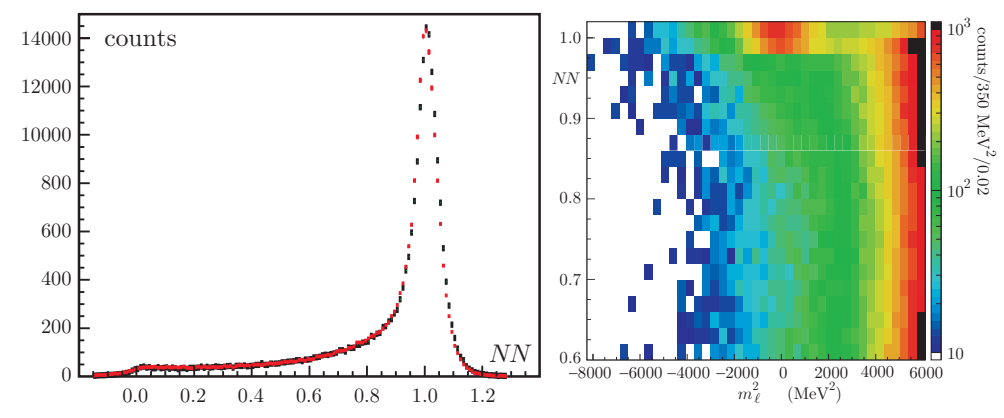

Figure 2: Left: Neural-network output, $N N$, for electrons of a $K_{L} \rightarrow \pi e v$ sample from data (black) and MC (red). Right: Data density in the $N N, m_{\ell}^{2}$ plane.

$m_{\ell}^{2}$ is shown in Fig. 2 right. A clear $K \rightarrow e v$ signal can be seen at $m_{\ell}^{2} \sim 0$ and $N N \sim 1$.

Some $32 \%$ of the events with a $K$ decay in the fiducial volume, have a reconstructed kink matching the required quality criteria and an EMC cluster associated to the lepton track; this holds for both $K_{e 2}$ and $K_{\mu 2}$. In the selected sample, the contamination from $K$ decays other than $K_{\ell 2}$ is negligible, as evaluated from MC. $R_{10}$, Eq. 2, is obtained without requiring the presence of the radiated photon. The number of $K \rightarrow e v(\gamma)$, is determined with a binned likelihood fit to the twodimensional $N N$ vs $m_{\ell}^{2}$ distribution. Distribution shapes for signal and $K_{\mu 2}$ background are taken from MC; the normalization factors for the two components are the only fit parameters. The fit has been performed in the region $-3700<m_{\ell}^{2}<6100 \mathrm{MeV}^{2}$ and $N N>0.86$. The fit region accepts $\sim 90 \%$ of $K \rightarrow e v(\gamma)$ events with $E_{\gamma}<10 \mathrm{MeV}$, as evaluated from MC. A small fraction of fitted $K \rightarrow e v(\gamma)$ events have $E_{\gamma}>10 \mathrm{MeV}$ : the value of this "contamination", $f_{\mathrm{DE}}$, is fixed in the fit to the expectation from simulation, $f_{\mathrm{DE}}=10.2 \%$. A systematic error related to this assumption is discussed in Sect. 2 .

We count $7064 \pm 102 K^{+} \rightarrow e^{+} v(\gamma)$ events and $6750 \pm 101 K^{-} \rightarrow e^{-} \bar{v}(\gamma), 89.8 \%$ of which have $E_{\gamma}<10 \mathrm{MeV}$. The signal-to-background correlation is $\sim 20 \%$ and the $\chi^{2} / \mathrm{ndf}$ is $113 / 112$ for $K^{+}$and 140/112 for $K^{-}$. Fig. 3 shows the sum of fit results for $K^{+}$and $K^{-}$projected onto the $m_{\ell}^{2}$ axis in a signal $(N N>0.98)$ and a background $(N N<0.98)$ region.

The number of $K_{\mu 2}$ events is obtained from a fit to the $m_{\ell}^{2}$ distribution. The fraction of background events under the muon peak is estimated from $\mathrm{MC}$ to be less than one per mil. We count $2.878 \times 10^{8}\left(2.742 \times 10^{8}\right) K^{+} \rightarrow \mu^{+} \nu(\gamma)\left(K^{-} \rightarrow \mu^{-} \bar{v}(\gamma)\right)$ events. The difference between $K^{+}$and $K^{-}$counts is due to $K^{-}$nuclear interactions in the material traversed.

\section{Efficiency and systematic errors evaluation}

The ratios of $K_{e 2}$ to $K_{\mu 2}$ efficiencies are evaluated with $\mathrm{MC}$ and corrected for possible differences between data and MC, using control samples. We evaluate data-MC corrections separately for each of the following analysis steps: decay point reconstruction (kink), quality cuts, clustercharged particle association. For each step, the correction is defined as the ratio of data and MC 

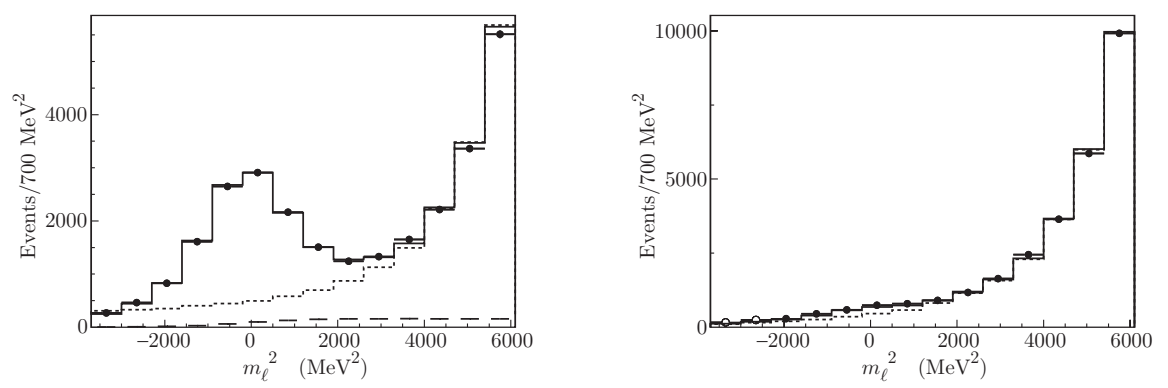

Figure 3: Fit projections onto the $m_{\ell}^{2}$ axis for $N N>0.98$ (left) and $N N<0.98$ (right), for data (black dots), MC fit (solid line), and $K_{\mu 2}$ background (dotted line). The contribution from $K_{e 2}$ events with $E_{\gamma}>10 \mathrm{MeV}$ is visible in the left panel (dashed line).

efficiencies measured on the control sample, each folded with the proper kinematic spectrum of $K_{e 2}$ (or $K_{\mu 2}$ ) events.

Decay point reconstruction efficiencies are evaluated using pure samples of $K_{\mu 2}$ and $K_{e 3}$; these are tagged by the identification of the two-body decay, $K_{\mu 2}$ or $K \rightarrow \pi \pi^{0}\left(K_{\pi 2}\right)$, of the other kaon and selected with tagging and EMC information only, without using tracking. The corrections to MC efficiencies range between 0.90 and 0.99 depending on the decay point position and on the decay angle. The simulation is less accurate in case of overlap between lepton and kaon tracks, and with decays occurring close to the inner border of the fiducial volume.

Samples of $K_{L}(e 3), K_{L}(\mu 3)$, and $K_{\mu 2}$ decays with a purity of $99.5 \%, 95.4 \%$, and $100.0 \%$ respectively, are used to evaluate lepton cluster efficiencies. These samples are selected using tagging and DC information only, without using calorimeter, see Refs. [9]. The efficiency has been evaluated as a function of the particle momentum separately for barrel and endcap. The correction to $\mathrm{MC}$ efficiencies ranges between 0.98 and 1.01 depending on the momentum and on the point of impact on the calorimeter. The trigger efficiency has been evaluated solely from data.

The absolute values of all of the systematic uncertainties on $R_{10}$ are listed in Table 1. All of the sources of systematic error are discussed below.

\begin{tabular}{|ccc|}
\hline & & $\delta\left(R_{10}\right) \times 10^{5}$ \\
\hline \multicolumn{2}{|c|}{ Statistical error } & 0.024 \\
\hline \multicolumn{2}{|c|}{ Systematic error } \\
Counting: & fit & 0.007 \\
& DE & 0.005 \\
Efficiency: & kink & 0.014 \\
& trigger & 0.009 \\
& $e, \mu$ cluster & 0.005 \\
Total systematic error & 0.019 \\
\hline
\end{tabular}

Table 1: Summary of statistical and systematic uncertainties on the measurements of $R_{10}$.

To minimize possible biases on $K_{e 2}$ event counting due to the limited knowledge of the momentum resolution, we used $K_{\mu 2}$ data to carefully tune the MC response on the tails of the $m_{\ell}^{2}$ distribution. This has been performed in sidebands of the $N N$ variable, to avoid bias due to the 
presence of $K_{e 2}$ signal. Similarly, for the $N N$ distribution the EMC response in the MC has been tuned at the level of single cell, using $K_{\ell 3}$ data control samples. Residual differences between data and $\mathrm{MC} K_{e 2}$ and $K_{\mu 2} N N$ shapes have been corrected by using the same control samples. Finally, to evaluate the systematic error associated with these procedures, we studied the variation of the results with different choices of fit range, corresponding to a change of overall purity from $\sim 75 \%$ to $\sim 10 \%$, for $K \rightarrow e v(\gamma)$ with $E_{\gamma}<10 \mathrm{MeV}$. A systematic uncertainty of $\sim 0.3 \%$ is derived by scaling the uncorrelated errors so that the reduced $\chi^{2}$ value equals unity (see also Table 1 ).

$K_{e 2}$ event counting is also affected by the uncertainty on $f_{\mathrm{DE}}$, the fraction of $K_{e 2}$ events in the fit region which are due to DE process. This error has been evaluated by repeating the measurement of $R_{10}$ with values of $f_{\mathrm{DE}}$ varied within its uncertainty, which is $\sim 4 \%$ according to our measurement of the $K_{e 2 \gamma}$ differential spectrum [7]. Since the $m_{\ell}^{2}$ distributions for $K_{e 2 \gamma}$ with $E_{\gamma}<10 \mathrm{MeV}$ and with $E_{\gamma}>10 \mathrm{MeV}$ overlap only partially, the associated fractional variation on $R_{10}$ is reduced: the final error due to DE uncertainty is $0.2 \%$ (Table 1 ).

Different contributions to the systematic uncertainty on $\varepsilon_{e 2} / \varepsilon_{\mu 2}$ are listed in Table 1. These errors are dominated by the statistics of the control samples used to correct the MC evaluations. In addition, we studied the variation of each correction with modified control-sample selection criteria. We found neglible contributions in all cases but for the kink and quality cuts corrections, for which the bias due to the control-sample selection and the statistics contribute at the same level.

The total systematic error is $\sim 0.8 \%$, to be compared with statistical accuracy at the level of $\sim 1 \%$.

\section{3. $R_{K}$ and lepton-flavor violation}

The number of $K \rightarrow e v(\gamma)$ events with $E_{\gamma}<10 \mathrm{MeV}$, the number of $K \rightarrow \mu v(\gamma)$ events, the ratio of $K_{e 2}$ to $K_{\mu 2}$ efficiencies and the measurement of $R_{10}$ are given in Table 2 for $K^{+}, K^{-}$and both charges combined. $K^{+}$and $K^{-}$results are consistent within the statistical error. The systematic uncertainty is common to both charges.

\begin{tabular}{|l|c|c|c|c|}
\hline & $\mathrm{N}\left(K_{e 2}\right)$ & $\mathrm{N}\left(K_{\mu 2}\right)$ & $\varepsilon_{e 2} / \varepsilon_{\mu 2}$ & $R_{10} \times 10^{5}$ \\
\hline$K^{+}$ & $6348 \pm 92 \pm 23$ & $2.878 \times 10^{8}$ & $0.944 \pm 0.003 \pm 0.007$ & $(2.336 \pm 0.033 \pm 0.019)$ \\
$K^{-}$ & $6064 \pm 91 \pm 22$ & $2.742 \times 10^{8}$ & $0.949 \pm 0.002 \pm 0.007$ & $(2.330 \pm 0.035 \pm 0.019)$ \\
$K^{ \pm}$ & $12412 \pm 129 \pm 45$ & $5.620 \times 10^{8}$ & $0.947 \pm 0.002 \pm 0.007$ & $(2.333 \pm 0.024 \pm 0.019)$ \\
\hline
\end{tabular}

Table 2: Number of $K_{e 2}$ and $K_{\mu 2}$ events, efficiency ratios and results for $R_{10}$ for $K^{+}, K^{-}$, and both charges combined; first error is statistical, second one is systematic.

To compare the $R_{10}$ measurement with the inclusive $R_{K}$ prediction from $\mathrm{SM}$, we take into account the acceptance of the $10 \mathrm{MeV}$ cut for IB, Eq. 3. We obtain: $R_{K}=\left(2.493 \pm 0.025_{\text {stat }} \pm\right.$ $\left.0.019_{\text {syst }}\right) \times 10^{-5}$, in agreement with SM prediction of Eq. 1. In the framework of MSSM with lepton-flavor violating (LFV) couplings, $R_{K}$ can be used to set constraints in the space of relevant parameters, using the following expression [3]:

$$
R_{K}=R_{K}^{S \mathrm{M}} \times\left[1+\left(\frac{m_{K}^{4}}{m_{H}^{4}}\right)\left(\frac{m_{\tau}^{2}}{m_{e}^{2}}\right)\left|\Delta_{R}^{31}\right|^{2} \tan ^{6} \beta\right],
$$


where $M_{H}$ is the charged-Higgs mass, $\Delta_{R}^{31}$ is the effective $e$ - $\tau$ coupling constant depending on MSSM parameters, and $\tan \beta$ is the ratio of the two Higgs superfields vacuum expectation values. The regions excluded at 95\% C.L. in the plane $M_{H}-\tan \beta$ are shown in Fig. 4 for different values of the effective LFV coupling $\Delta_{R}^{31}$.

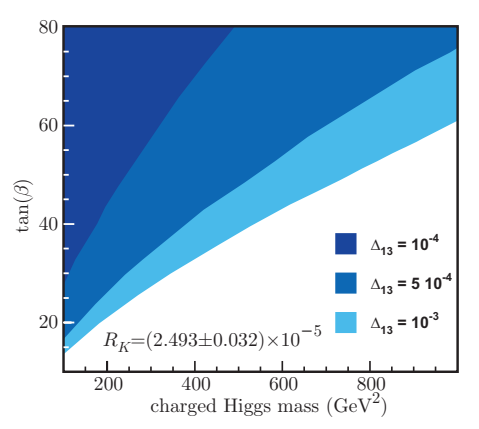

Figure 4: Excluded regions at $95 \%$ C.L. in the plane $M_{H^{-}} \tan \beta$ for $\Delta_{R}^{31}=10^{-4}, 5 \times 10^{-3}, 10^{-3}$.

\section{Conclusions}

We have performed a comprehensive study of the process $K_{e 2 \gamma}$. We have measured the ratio of $K_{e 2 \gamma}$ and $K_{\mu 2}$ widths for photon energies smaller than $10 \mathrm{MeV}$, without photon detection requirement. We find: $R_{10}=\left(2.333 \pm 0.024_{\text {stat }} \pm 0.019_{\text {stat }}\right) \times 10^{-5}$. From this result we derive the inclusive ratio $R_{K}$ to be compared with the SM prediction: $R_{K}=\left(2.493 \pm 0.025_{\text {stat }} \pm 0.019_{\text {syst }}\right) \times 10^{-5}$, in excellent agreement with the SM prediction $R_{K}=(2.477 \pm 0.001) \times 10^{-5}$. Our result improves the accuracy with which $R_{K}$ is known by a factor of 5 with respect to the present world average and allows severe constraints to be set on new physics contributions in the MSSM with lepton flavor violating couplings.

\section{References}

[1] W.J. Marciano and A. Sirlin, Phys. Rev. Lett. 71 (1993) 3629; M. Finkemeier, Phys. Lett. B 387 (1996) 391.

[2] V. Cirigliano and I. Rosell, Phys. Rev. Lett. 99 (2007) 231801.

[3] A. Masiero, P. Paradisi and R. Petronzio, JHEP 0811 (2008) 042

[4] J. Bijnens, G. Colangelo, G. Ecker and J. Gasser, [hep-ph/9411311].

[5] J. Heintze et al., Nucl. Phys. B 149 (1979) 365.

[6] N. Cabibbo, Phys. Rev. Lett. 10 (1963) 531.

[7] See M. Moulson contribution in these proceedings.

[8] C. Gatti, Eur. Phys. J. C 45, 417 (2006).

[9] F. Ambrosino et al., KLOE Coll., Phys. Lett., B 632 (2006) 43-50; F. Ambrosino et al., KLOE Coll., Phys. Lett. B 636, 166 (2006); F. Ambrosino et al., KLOE Coll., JHEP 12 (2007) 105

[10] M. Antonelli et al. arXiv:0801.1817 [hep-ph]. 